\title{
Unconventional Superconductivity, Spontaneous Time-Reversal Symmetry Breaking and Lowering of Kinetic Energy in Doped, Short-Range Antiferromagnets
}

\author{
P. Wróbel ${ }^{a, c}$, A. MACiA̧G ${ }^{a, *}, \mathrm{R} . \mathrm{EdeR}^{b}, \mathrm{P} \cdot \mathrm{FuldE}^{c}$ \\ AND R. MICNAS ${ }^{d}$ \\ ${ }^{a}$ Institute for Low Temperature and Structure Research \\ P.O. Box 1410, 50-950 Wrocław 2, Poland \\ ${ }^{b}$ Forschungszentrum Karlsruhe, IFP \\ P.O. Box 3640, 76021 Karlsruhe, Germany \\ ${ }^{c}$ Max Planck Institute for the Physics of Complex Systems \\ 01187 Dresden, Germany \\ ${ }^{d}$ Adam Mickiewicz University, Institute of Physics \\ Umultowska 85, 61-614 Poznań, Poland
}

\begin{abstract}
In this paper we focus on the anomalous temperature dependence of the in-plane conductivity and symmetry mixing of the superconducting order parameter observed in various experiments on cuprates. We show that the one-band Hubbard model is not capable of describing the physics of cuprates because the kinetic energy is lowered in this model in the superconducting state, which contradicts experimental observations. The proper model to investigate doped, short-range antiferromagnets is the $t-J$ model, for which our results agree with experiments. We analyze a spin polaron model, that is an effective model for a doped antiferromagnet. In the framework of this model we also study the superconducting order-parameter symmetry-mixing phenomenon. We show that the expected mixing of $d$-wave symmetry with $p$-wave symmetry takes place in the superconducting order-parameter at a finite value of the doping parameter. This symmetry mixing brakes the time-reversal symmetry.
\end{abstract}

PACS numbers: 74.20.Mn, 71.10.Fd

*corresponding author; e-mail: maciag@int.pan.wroc.pl 


\section{Introduction}

Irrefutable evidence for unconventional superconductivity observed in doped short-range antiferromagnets (AFs) has been gathered during the last few years. It has been observed [1] that the spectral weight of the optical conductivity is shifted towards lower energies below $T_{\mathrm{c}}$ and in the pseudogap region, which indicates that the lowering of the kinetic energy plays an important role in pairing. The integrated spectral weight is defined as

$$
W=\int_{0}^{\omega_{m}} \sigma_{1, \delta}(\omega, T) \mathrm{d} \omega,
$$

where $\sigma_{1, \delta}(\omega, T)$ is the optical conductivity and $\omega_{m}$ is a cut-off frequency. When $\omega_{m}=\infty$ the spectral weight should be conserved, which is known as the optical sum rule. That rule should, in principle, be exhausted at the cut-off frequency, which is determined by an upper bound for the spectrum excitations mediating pairing. For conventional superconductors, for which this sum rule is preserved, the upper bound may be set roughly at the energy $4 \Delta$, where $\Delta$ is the superconducting (SC) gap. Assuming a similar pairing mechanism in $d$-wave cuprates, where the maximum gap value is $25 \mathrm{meV}$, the cut-off frequency value should be $\sim 0.1 \mathrm{eV}$ to exhaust the optical sum rule. Nevertheless, it has been observed that for underdoped Bi-2212 this cut-off frequency should be set at $2 \mathrm{eV}$ to exhaust the sum rule [2].

In the next section of this paper we will argue that the operator which represents the kinetic-like energy and whose thermodynamic average is measured by the optical integral, depends on the upper limit of this integral. If the upper limit for the integration lies below the charge excitation energy $U$, the kineticlike energy should be defined by the hopping term in the $t-J$ model $(t J \mathrm{M})$. The measurements indicate that this quantity is lowered in the SC state [1]. If the span of the optical integral covers charge excitations, a proper definition of the true kinetic energy relates it to the energy of hopping in the Hubbard model (HM). In this case, the experimentally derived integral is conserved in the SC state and no lowering of the kinetic energy is expected [2].

Another characteristic feature of unconventional superconductivity is the mixing of pairing symmetries and breaking of the time-reversal symmetry. There is no doubt that the $d_{x^{2}-y^{2}}$ symmetry plays a dominating role in the SC state of cuprates. Notwithstanding this, a strong tendency towards development of a secondary gap component of different symmetry appears in high $T_{\mathrm{c}}$ cuprate superconductors. Both in the magnetic field and in Ni-doped systems an anomaly in the thermal conductivity is observed in $\mathrm{Bi}_{2} \mathrm{Sr}_{2} \mathrm{CaCu}_{2} \mathrm{O}_{8}$ superconductors [3]. The formation of that anomaly may be attributed to the mixing of pairing symmetries and to the breaking of time-reversal symmetry [4]. An admixture of a symmetry

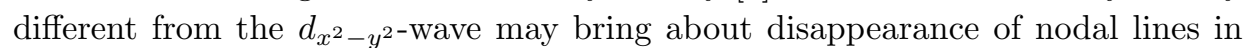
the gap along the diagonals of the Brillouin zone (BZ). A locally nodeless order 
parameter [5] accounts for the enhanced density of states observed in the vortex core in tunneling measurements. A mixed singlet triplet paring $d+\mathrm{i} p$ has been invoked [6] to account for the time-reversal breaking in the pseudogap phase and in the $\mathrm{SC}$ phase in $\mathrm{Bi}_{2} \mathrm{Sr}_{2} \mathrm{CaCu}_{2} \mathrm{O}_{8+\delta}$. We are going to demonstrate in this paper that such mixing may be generated in a microscopic model, i.e. the $t J \mathrm{M}$ [7]. We will present the spin polaron model (SPM), which represents the physics of low-energy excitations in the $t J \mathrm{M}$ in the doping region, where at least short-range $\mathrm{AF}$ correlations exist. This Hamiltonian is defined in the language of spin polarons, which are temporary localized holes dressed with a cloud of spin fluctuations. In the SPM the nature of propagation of quasiparticles and interaction between them is determined by a tendency to restore the local AF order. It is known that two holes in the $t J \mathrm{M}$ form bound states with $d_{x^{2}-y^{2}}$ or $p$-wave symmetry. The $d$-wave bound state has a lower energy and is the ground state. We will demonstrate that in the SPM the mixing of the $d$-wave symmetry with the $p$-wave symmetry takes place in the SC order parameter at a finite value of the doping parameter, which lies at the applicability edge of the SPM. We will show that this kind of symmetry mixing removes nodal lines in the gap along the diagonal of the BZ, which may account for the enhanced density of states observed in the vortex core.

\section{Spectral weight and the kinetic energy}

The task of formulating a universal model capable of describing simultaneously all the experimental aspects of cuprates seems elusive. Nevertheless, it is believed that some general understanding of the SC state in doped AF may be gained from analysis of minimal models for such systems, e.g. the HM and the $t J \mathrm{M}$, to which the HM may reduce in the limit of strong correlations.

For models in which electrons may only hop to the nearest neighbor (NN) sites, the optical integral is proportional with the overall minus sign to the kinetic energy

$$
W\left(T, \omega_{m}\right)=\int_{0}^{\omega_{m}} \mathrm{~d} \omega \sigma_{1, \delta}(\omega, T) \propto\left\langle-T_{\delta}\right\rangle .
$$

The answer to the question as to which part of the energy is measured by the optical integral depends on where an upper limit for it is set. The HM which is supposed to describe processes up to the energy scale $U$, is $H=H_{\mathrm{hHM}}+H_{U}, H_{\mathrm{hHM}}=$ $-t \sum_{\langle i, j\rangle, \sigma}\left(c_{i, \sigma}^{\dagger} c_{j, \sigma}+\right.$ h.c. $), H_{U}=U \sum_{i} n_{i, \uparrow} n_{i, \downarrow}$, where $H_{\mathrm{hHM}}$ is the hopping term which defines the kinetic energy at the level of the HM. The $t J \mathrm{M}$ may be derived from the HM by means of a unitary transformation $[7,8]$ which decouples higher energy processes from lower energy processes. To achieve this aim we chose a simple procedure based on the standard perturbation theory. The bare Hamiltonian $H=H_{0}+H_{1}$ consists of an unperturbed part $H_{0}$ and a small perturbation $H_{1}$. We assume that the degenerate subspace $\boldsymbol{\Phi}^{(0)}$ is spanned by eigenvectors $\left|\phi_{0}^{(0)}\right\rangle$ of $H_{0}$, with eigenvalue $E^{(0)}$. The orthogonal subspace $\boldsymbol{\Psi}^{(0)}$ is spanned by eigenvectors 
$\left|\psi_{m}^{(0)}\right\rangle$ with eigenvalues $E_{m}^{(0)}$ of $H_{0}$ which are higher than $E^{(0)}$ by at least a finite gap value. When the perturbation is adiabatically switched on, $\boldsymbol{\Phi}^{(0)}$ and $\boldsymbol{\Psi}^{(0)}$ will evolve into orthogonal eigenspaces of $H, \boldsymbol{\Phi}$, and $\boldsymbol{\Psi}$ with defining property, $H \boldsymbol{\Phi}=\boldsymbol{\Phi}$ and $H \boldsymbol{\Psi}=\boldsymbol{\Psi}$. For the HM in the large $U$ limit, the hopping term $H_{\mathrm{hHM}}$ is a perturbation. $\boldsymbol{\Phi}^{(0)}$ is spanned by functions which represent states with no doubly occupied sites, and $\boldsymbol{\Psi}^{(0)}$ is an orthogonal subspace spanned by functions which represent states with some double occupied site. $H_{1}=H_{\mathrm{hHM}}$ may couple a state from subspace $\boldsymbol{\Phi}^{(0)}$ with a state from subspace $\boldsymbol{\Psi}^{(0)}$. The unitary transformation $\hat{U}$, which we are looking for, transforms $\boldsymbol{\Phi}^{(0)}$ into $\boldsymbol{\Phi}$. The kinetic energy $H_{\mathrm{hHM}}$ defined for the HM after a unitary transformation to the level of the $t J \mathrm{M}$ is represented by the operator

$$
\begin{aligned}
H_{\mathrm{hHM}}^{(t J)} & =P_{0} \hat{U}^{\dagger} H_{\mathrm{hHM}} \hat{U} P_{0} \\
= & -t \sum_{\langle i, j\rangle, \sigma}\left(\tilde{c}_{i, \sigma}^{\dagger} \tilde{c}_{j, \sigma}+\text { h.c. }\right)+2 J \sum_{\langle i, j\rangle}\left(S_{i} S_{j}-\frac{\tilde{n}_{i} \tilde{n}_{j}}{4}\right),
\end{aligned}
$$

where $\tilde{c}_{i, \sigma}=c_{i, \sigma}\left(1-n_{i, \sigma}\right), J=4 t^{2} / U, P_{0}=P_{0}^{\dagger}$ is a projector on the space $\boldsymbol{\Phi}^{(0)}$, and the sum over $\langle i, j\rangle$ stands for summation over NN sites. We neglect the hopping between the second and third NN in the second term. The formula for the transformed interaction term is

$$
H_{U}^{(t J)}=P_{0} \hat{U}^{\dagger} H_{U} \hat{U} P_{0}=-J \sum_{\langle i, j\rangle}\left(S_{i} S_{j}-\frac{\tilde{n}_{i} \tilde{n}_{j}}{4}\right),
$$

where we again neglect the hopping between the second and third NN sites. The Hamiltonian of the $t J \mathrm{M}$ which in the space with no doubly occupied sites, represents the full Hamiltonian $H^{(t J)}=P_{0} \hat{U}^{\dagger} H \hat{U} P_{0}$, is given by the sums $H^{(t J)}=H_{\mathrm{hHM}}^{(t J)}+H_{U}^{(t J)}$ or $H^{(t J)}=H_{\mathrm{h} t J}+H_{J}$. Both the transformed Hamiltonian $H^{(t J)}$ and the transformed kinetic energy operator $H_{\mathrm{hHM}}^{(t J)}$ contain terms related to the hopping and the exchange energy in the $t J \mathrm{M}$. Thus, if the system manages somehow to lower simultaneously the hopping energy and the exchange energy at the level of $t J \mathrm{M}$, a lowering of the total and kinetic energies defined at the level of the HM is an obvious consequence. Now we can find which part of the energy is measured by the optical integral depending on, where its upper limit is set. When the upper limit for integration lies above the charge transfer gap we use the kinetic energy of the HM represented at the level of the $t J \mathrm{M}$

$$
\begin{gathered}
W\left(T, U^{+}\right)=\int_{0}^{U^{+}} \sigma_{1, \delta}(\omega, T) \mathrm{d} \omega \propto-\left\langle H_{\mathrm{hHM}}\right\rangle=\left\langle H_{\mathrm{hHM}}^{(t J)}\right\rangle \\
=-\left\langle-t \sum_{\langle i, j\rangle, \sigma}\left(\tilde{c}_{i, \sigma}^{\dagger} \tilde{c}_{j, \sigma}+\text { h.c. }\right)+2 J \sum_{\langle i, j\rangle}\left(S_{i} S_{j}-\frac{\tilde{n}_{i} \tilde{n}_{j}}{4}\right)\right\rangle .
\end{gathered}
$$

When the cut-off frequency is set below the charge excitation energy $U$, the spectral weight measures the average of the hopping energy in the $t J \mathrm{M}$, 


$$
\begin{gathered}
W\left(T, U^{-}\right)=\int_{0}^{U^{-}} \sigma_{1, \delta}(\omega, T) \mathrm{d} \omega \propto-\left\langle H_{\mathrm{h} t J}\right\rangle \\
=-\left\langle-t \sum_{\langle i, j\rangle, \sigma}\left(\tilde{c}_{i, \sigma}^{\dagger} \tilde{c}_{j, \sigma}+\text { h.c. }\right)\right\rangle .
\end{gathered}
$$

We are going to evaluate integrals (5) and (6) in the framework of the SPM.

\section{The effective model, the superconducting state and the kinetic energy}

In this section we will not construct the full spin polaron Hamiltonian, but explain the two most important processes which contribute to it. For detailed information we refer to previous papers [8,9]. A basic assumption underlying the $\mathrm{SPM}$ is that the AF correlation length is longer than the distance at which a hole moving in the AF background gets effectively localized in an AF spin medium. We will show this "quasi"-localization on the example of a hole moving in the Néel state. Let us first consider a hole created in the Néel state, Fig. 1a. This hole spoils the local AF arrangement by breaking the NN AF bonds. The potential-like energy is related to the Ising term, $J \sum_{\langle i, j\rangle}\left(S_{i}^{z} S_{j}^{z}-\tilde{n}_{i} \tilde{n}_{j} / 4\right)$. The hopping hole shifts spins and creates defects in the AF background. These processes are depicted in Fig. 1a-c and give rise to the increase in potential-like energy. Frustrated spins which the hole has left in its trail tend to localize it and act on the hole as a kind of string. Since $t \gg J$ the probability of a next hop is higher than that of any process mediated by the exchange term $[8,9]$. Thus, in the lowest order approximation we may assume that the trapped hole will only oscillate in the potential well related to strings formed by defected spins. On the other hand, the action of the term $\frac{J}{2} \sum_{\langle i, j\rangle}\left(S_{i}^{+} S_{j}^{-}+\right.$h.c. $)$in the Hamiltonian may release the trapped hole. This term flips antiparallel spins at NN sites and restores AF arrangements, Fig. 1d. The

\section{$\begin{array}{lllll}1 & 1 & 1 & 1 & 1\end{array}$ InOgi $\downarrow$ i}

(a)

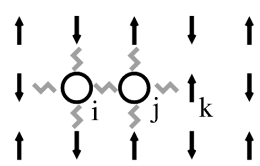

(e)

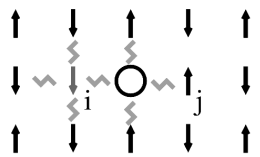

(b)

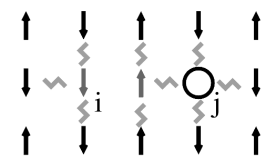

(c)

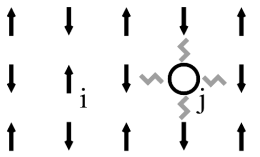

(d)

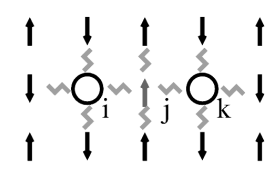

(f)

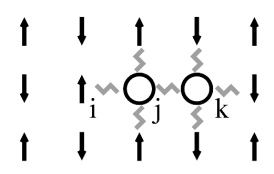

(g)

Fig. 1. Some processes which give rise to some hopping and interaction terms in the Hamiltonian. 
whole process giving rise to the motion of the spin polaron from site $i$ to site $j$ is presented in the sequence of Fig. 1a-d. We notice that the hopping of the spin polarons is mediated by the hopping and exchange interaction at the level of the $t J \mathrm{M}$. The hopping of spin polarons appears in the SPM as a term $\propto h^{\dagger} h$, where $h^{\dagger}$ and $h$ are spin polaron creation and annihilation operators, respectively [2]. Two holes created at distant sites may be treated as two independent spin polarons. Two holes created on NN sites act on each other by restricting their freedom of motion. Hopping of one of the holes spoils the AF background, Fig. 1f, which can be repaired by moving the other hole in the same direction, Fig. 1g. This process gives rise to a two-body attractive interaction term $\propto h^{+} h^{+} h h$ in the SMP $[8,9]$. We notice that the contribution to the interaction between spin polarons is mediated by hopping at the level of the $t J \mathrm{M}$ only.

The full SPM Hamiltonian [9] describing pairing in the real space between spin polarons at distances not greater than three lattice spacings is used in this paper. Now we are searching for an answer to the question as to whether the SC state is formed in the SPM and, if so, what the symmetry of that state is. We take into account five irreducible representations of the point group of the squarelattice symmetry: $A_{1}(s), A_{2}(g), B_{1}\left(d_{x^{2}-y^{2}}\right), B_{2}\left(d_{x y}\right)$, and $E\left(p_{x(y)}\right)$. In order to achieve this goal, we embark on the Hartree-Fock analysis of the Hamiltonian represented in terms of creation and annihilation operators to which a Fourier transformation has been applied. Since the system which we analyze is two dimensional, we concentrate on the case of vanishing temperature $T=0$, for which the SC state may exist. It turns out that at a given charge concentration only stable solutions are a pure $d_{x^{2}-y^{2}}$-wave state or a mixture of $d_{x^{2}-y^{2}}+\mathrm{i} p_{x(y)}$-wave symmetries. The admixture of $p$-wave symmetry appears in the order parameter for relatively high hole-doping values, which lie near the border of applicability for the spin polaron approach. Due to the admixture of the $p_{x}$-wave in the gap function, the node, which exists when the symmetry is a pure $d$-wave, disappears for a doping parameter higher than the limiting value $\delta \simeq 0.12$. By means of the SPM we have also evaluated the integrals (5) and (6). It turns out that both the kinetic energy in the HM and the hopping energy in the $t J \mathrm{M}$ decrease in the $\mathrm{SC}$ state. The lowering of the kinetic energy in the HM contradicts experiments, which show that the integrated spectral weight (1) with the cut-off frequency put above the charge transfer gap, does not change in the SC state.

\section{Conclusions}

The analysis presented in this paper allows one to state that the single-band Hubbard model is not capable of describing excitations across the charge transfer gap in cuprates. Lowering of the kinetic energy in that model in the SC state contradicts experimental observations [2]. On the other hand, experimental observations may be interpreted in terms of lowering of the hopping energy in 
the $t J \mathrm{M}$ in the $\mathrm{SC}$ state of the doped $\mathrm{AF}$, which may be attributed to the fact that hopping in the $t J \mathrm{M}$ plays the role of interaction for some effective quasi-particles, i.e. spin polarons. We have also observed that in the SPM the SC ground state appears with $d_{x^{2}-y^{2}}$-wave symmetry of the $\mathrm{SC}$ order parameter for a small value of the doping parameter. The SC ground state with mixed $d_{x^{2}-y^{2}}+\mathrm{i} p_{x(y)}$-wave symmetries of the SC order parameter occurs at a finite value of the doping parameter, which lies at the applicability verge of the SPM. The mixing of different symmetries accounts for some experiments indicating disappearance of nodal lines in the cuprates. A phase shift by $\pi$ between components of mixing symmetries leads to spontaneous time-reversal symmetry breaking. The spin of the $p$-wave component of the order parameter defined in the real space is perpendicular to the direction of the local sublattice magnetization.

\section{Acknowledgment}

P.W. acknowledges partial support by the State Committee for Scientific Research (Poland) under contract No 2P03B00925.

\section{References}

[1] H.J.A. Molengraaf, C. Presura, D. van der Marel, P.H. Kes, M. Li, Science 295, 2239 (2002).

[2] A.F. Santander-Syro, R.P.S.M. Lobo, N. Bontemps, Z. Konstantovic, Z.Z. Li, H. Raffy, preprint cond-mat/0111539, Phys. Rev. Lett. 88, 097005 (2002).

[3] K. Krishana, N.P. Ong, Q. Li, G.D. Gu, N. Koshizuka, Science 277, 83 (1997); R. Movshovich, M.A. Hubbard, M.B. Salamon, A.V. Balatsky, R. Yoshizaki, J.L. Sarrao, M. Jaime, Phys. Rev. Lett. 80, 1968 (1998).

[4] R.B. Laughlin, Phys. Rev. Lett. 80, 5188 (1998).

[5] M. Franz, Z. Tešanović, Phys. Rev. Lett. 80, 4763 (1998).

[6] R.P. Kaur, D.F. Agterberg, Phys. Rev. B 68, 100506 (2003).

[7] K.A. Chao, J. Spalek, A.M. Oles, Phys. Rev. B 18, 3453 (1978).

[8] P. Wróbel, R. Eder, P. Fulde, J. Phys. Condens. Matter 15, 6599 (2003).

[9] P. Wróbel, R. Eder, R. Micnas, J. Phys. Condens. Matter 15, 2755 (2003). 